\title{
Amplification limit of weak measurements: a variational approach
}

\author{
Shengshi Pang ${ }^{1} *$ Todd A. Brun ${ }^{1}$ S Shengjun $\mathrm{Wu}^{2,3}$ 团 and Zeng-Bing Chen $2 \S$ \\ ${ }^{1}$ Department of Electrical Engineering, University of Southern California, Los Angeles, California 90089, USA \\ ${ }^{2}$ Hefei National Laboratory for Physical Sciences at Microscale and Department of Modern Physics, \\ University of Science and Technology of China, Hefei, Anhui 230026, China and \\ ${ }^{3}$ Kuang Yaming Honors School, Nanjing University, Nanjing 210093, China
}

\begin{abstract}
Post-selected weak measurement has been widely used in experiments to observe weak effects in various physical systems. However, it is still unclear how large the amplification ability of a weak measurement can be and what determines the limit of this ability, which is fundamental to understanding and applying weak measurements. The limitation of the conventional weak value formalism for this problem is the divergence of weak values when the pre- and post-selections are nearly orthogonal. In this paper, we study this problem by a variational approach for a general Hamiltonian $H_{\text {int }}=g A \otimes \Omega \delta\left(t-t_{0}\right), g \ll 1$. We derive a general asymptotic solution, and show that the amplification limit is essentially independent of $g$, and determined only by the initial state of the detector and the number of distinct eigenvalues of $A$ or $\Omega$. An example of spin- $\frac{1}{2}$ particles with a pair of Stern-Gerlach devices is given to illustrate the results. The limiting case of continuous variable systems is also investigated to demonstrate the influence of system dimension on the amplification limit.

PACS numbers: 03.65.Ta, 03.65.Ca, 03.67.Ac, 42.50.-p
\end{abstract}

\section{INTRODUCTION}

The von Neumann projective measurement model is well known theory for standard quantum measurements, in which the readings of a measurement are the eigenvalues of an observable, and the system is projected into the corresponding eigenstate of the observable. To realize such an ideal measurement, the spread width of the detector's wave functions must be sufficiently narrow, or the interaction between the system and the detector must be sufficiently strong, so that the detector's final statestranslated by different eigenvalues of the observable - can be distinguished with high probability.

In contrast to von Neumann measurements, weak measurements (coined by Aharonov, Albert, and Vaidman in 1988 [1]) exploit the opposite conditions: the initial detector state has a very wide spread, or the measurement strength is ultra-weak. Such a weak measurement makes the detector's final states, translated by different eigenvalues of the system observable, significantly overlap with each other. And a further step of this protocol, postselection, superposes these states. Interference between them can dramatically change the original state of the detector (not only by a translation). A remarkable effect induced by this interference is that the output from the postselected detector can be much larger than the eigenvalue spectrum of the system observable, due to the coherence in the superposed state of the detector canceling the major part of the original detector wave function [2].

This striking difference from standard von Neumann

\footnotetext{
* shengshp@usc.edu

$\dagger$ tbrun@usc.edu

$\ddagger$ sjwu@nju.edu.cn

$\S$ zbchen@ustc.edu.cn
}

measurements makes weak measurement particularly useful to amplify small physical quantities. Experiments have successfully realized the amplification of many different physical effects by postselected weak measurements, including the spin Hall effect of light [3, 4], optical beam deflection [5, 6], optical frequency shift [7], optical phase shift [8, 9], temperature shift [10], longitudinal velocities [11, etc. More experimental protocols have been proposed [12 20]. Moreover, weak measurements have been realized on various physical systems besides optical systems, including SQUIDs [21, 22] and NMR [23], among others.

Despite the considerable existing research on the theory of weak measurements, and their increasing application in experiments, a fundamental problem is still unclear: what is the ultimate limit of amplification in a postselected weak measurement? Usually, weak measurements are studied in the weak value formalism (see [24] for a general framework, and [25, 26] for reviews). However, in the weak value formalism, the amplification of a weak measurement can be infinitely large if the inner product between the pre- and postselections of the system is sufficiently small. From a practical view, this is obviously impossible. The root of this contradiction is that the weak value formalism is valid only when the amplification is small, since it is a first-order approximation theory that works only when the response of the detector is linear in the signal. When the amplification is too strong, the response of the detector to the signal becomes nonlinear, so the weak value formalism breaks down. It cannot give a valid result for the limit of amplification in a postselected weak measurement.

The significance of this problem is manifold: (1) it determines clearly to what extent postselected weak measurements can amplify small signals in practical experiments, thus revealing the limits of the quantum advan- 
tage in this task; (2) it can show what determines the amplification limit of a weak measurement, thus providing guidance for designing experiments; (3) it goes beyond the limitation of the weak value formalism, so its result will be an important supplement to the current knowledge of weak measurement. Given the wide application of weak measurements in many different branches of physics, the solution of this problem will be broadly useful to the physics community.

Despite the importance of this problem, few results have been known to date, and a complete solution is still missing. Numerical upper bounds were observed for some cases in [24, 27]. Certain special cases were studied with specific assumptions on the observable $A$ (e.g., $A^{2}=I$ in [28, 29]), or on the detector states (e.g., a qubit system with a Gaussian detector in [30]). Orthogonal and asymptotically orthogonal pre- and postselections were considered in [31]. An optimal detector for a given experimental setup was provided in 32]. In a more recent paper [33], a refined weak value method was attempted; but it is still not sufficient to give a rigorous solution without including higher order weak values - particularly when the dimension of the system or the detector is high - since higher order weak values can dominate over the lowest order weak value. (This has been verified by weak measurements with OAM pointer states [34].)

In this paper, we fill this gap by exploiting a variational approach to study the problem in a rigorous way. We derive a general analytical solution for a weak coupling Hamiltonian $H_{\text {int }}=g A \otimes \Omega \delta\left(t-t_{0}\right), g \ll 1$, and reveal the surprising property that the solution is independent of the coupling strength $g$ when $g \ll 1$, depending only on the initial state of the detector and the dimension of the system or the detector, whichever is less (if $A$ and $\Omega$ are nondegenerate). This is in marked contrast to the weak value formalism. For degenerate $A$ or $\Omega$, the degeneracy will decrease the amplification limit, which depends on the number of distinct eigenvalues. The results are illustrated in detail by an example of spin- $\frac{1}{2}$ particles passing through a pair of Stern-Gerlach devices. We also consider continuous variable systems as a limiting example, to show how the dimension of the system can significantly influence the amplification limit, which also is missed by the weak value formalism.

\section{PRELIMINARY: THE WEAK VALUE FORMALISM}

We start by revisiting the weak value formalism. Suppose the initial state of the system is $\left|\Psi_{i}\right\rangle$, the postselected state is $\left|\Psi_{f}\right\rangle$, the initial state of the detector is $|\Upsilon\rangle$, and the interaction Hamiltonian between the system and the detector is

$$
H_{\mathrm{int}}=g A \otimes \Omega \delta\left(t-t_{0}\right)
$$

Let $\hbar=1$. The final state of the detector after the interaction followed by the postselection is $\left|\Upsilon_{f}\right\rangle=$
$\left\langle\Psi_{f}|\exp (-\mathrm{i} g A \otimes \Omega)| \Psi_{i}\right\rangle|\Upsilon\rangle$, where $\left|\Upsilon_{f}\right\rangle$ is unnormalized. If we measure an observable $M$ on the final detector state, the detector will have a shift of $\frac{\left\langle\Upsilon_{f}|M| \Upsilon_{f}\right\rangle}{\left\langle\Upsilon_{f} \mid \Upsilon_{f}\right\rangle}-\langle\Upsilon|M| \Upsilon\rangle$ in the expected value of $M$.

When $g$ is sufficiently small, the final state of the detector is approximately $\exp \left(-\mathrm{i} g A_{w} \Omega\right)|\Upsilon\rangle$, where

$$
A_{w}=\frac{\left\langle\Psi_{f}|A| \Psi_{i}\right\rangle}{\left\langle\Psi_{f} \mid \Psi_{i}\right\rangle}
$$

is called the weak value. It can be derived that the average output of the detector is

$$
\begin{aligned}
& g \operatorname{Im} A_{w}(\langle\Upsilon|\{\Omega, M\}| \Upsilon\rangle-2\langle\Upsilon|\Omega| \Upsilon\rangle\langle\Upsilon|M| \Upsilon\rangle) \\
& +\mathrm{i} g \operatorname{Re} A_{w}\langle\Upsilon|[\Omega, M]| \Upsilon\rangle
\end{aligned}
$$

The second term of Eq. (3) is real because the average of a commutator must be imaginary.

One can see from (3) that the average output of a weak measurement approximately amplifies $g$ by the real or imaginary part of the weak value. This is the basis of all weak measurement amplification protocols. It is worth mentioning that Ref. 35] demonstrated the roles of the real and imaginary parts of the weak value in the position or momentum shift of the detector in a postselected weak measurement. Eq. (3) is a generalization of those results to an arbitrary observable $M$ on the detector, and when $[\Omega, M]=0$ or i, (3) reduces to the results of [35].

An obvious shortcoming of the weak value formalism is that when $\left\langle\Psi_{f} \mid \Psi_{i}\right\rangle \rightarrow 0, A_{w} \rightarrow \infty$, implying that the output of a weak measurement could be infinite, which is impossible in practice. This issue is rooted in the first order approximation in deriving the weak value formalism, so it is not a proper tool to study the amplification limit of a weak measurement.

\section{AMPLIFICATION LIMIT: A VARIATIONAL APPROACH}

In the following, we shall show a variational approach to this problem that can avoid the divergence of the weak value formalism and give a valid result for the amplification limit.

Let's define a shift operator $\Delta M=M-\langle\Upsilon|M| \Upsilon\rangle$. The average shift of the detector is

$$
\langle\Delta M\rangle=\frac{\left\langle\Upsilon_{f}|\Delta M| \Upsilon_{f}\right\rangle}{\left\langle\Upsilon_{f} \mid \Upsilon_{f}\right\rangle}
$$

When we choose different pre- and postselections of the system, the detector will give different outputs at the end of the measurement. Intuitively, the shift of the detector should be bounded. Our goal is to find the maximum $\langle\Delta M\rangle$ over all possible pre- and postselections. This maximum is the amplification limit. 
When $\langle\Delta M\rangle$ attains an extremal value $\langle\Delta M\rangle_{\mathrm{e}}$, its variation with respect to $\left|\Upsilon_{f}\right\rangle$ is zero:

$$
\begin{aligned}
\delta\langle\Delta M\rangle_{\mathrm{e}} & =\frac{1}{\left\langle\Upsilon_{f} \mid \Upsilon_{f}\right\rangle}\left(\left(\delta\left\langle\Upsilon_{f}\right|\right)\left(\Delta M\left|\Upsilon_{f}\right\rangle-\left|\Upsilon_{f}\right\rangle\langle\Delta M\rangle_{\mathrm{e}}\right)\right. \\
& \left.+\left(\left\langle\Upsilon_{f}\right| \Delta M-\langle\Delta M\rangle_{\mathrm{e}}\left\langle\Upsilon_{f}\right|\right)\left(\delta\left|\Upsilon_{f}\right\rangle\right)\right)=0
\end{aligned}
$$

according to the variational principle. Since $\left|\Upsilon_{f}\right\rangle$ is determined by $\left|\Psi_{i}\right\rangle$ and $\left|\Psi_{f}\right\rangle$, the variation of $\left|\Upsilon_{f}\right\rangle$ can be expressed in terms of the variations of $\left|\Psi_{i}\right\rangle$ and $\left|\Psi_{f}\right\rangle$ :

$$
\begin{aligned}
\delta\left|\Upsilon_{f}\right\rangle & =\left(\delta\left\langle\Psi_{f}\right|\right) \exp (-\mathrm{i} g A \otimes \Omega)\left|\Psi_{i}\right\rangle|\Upsilon\rangle \\
& +\left\langle\Psi_{f}\right| \exp (-\mathrm{i} g A \otimes \Omega)\left(\delta\left|\Psi_{i}\right\rangle\right)|\Upsilon\rangle
\end{aligned}
$$

Thus, the variation of $\langle\Delta M\rangle_{\mathrm{e}}$ becomes

$$
\begin{aligned}
& \delta\langle\Delta M\rangle_{\mathrm{e}} \\
& =\langle\Upsilon|\left\langle\Psi _ { i } \left|\exp (\mathrm{i} g A \otimes \Omega)\left(\Delta M\left|\Upsilon_{f}\right\rangle-\left|\Upsilon_{f}\right\rangle\langle\Delta M\rangle_{\mathrm{e}}\right)\left(\delta\left|\Psi_{f}\right\rangle\right)\right.\right. \\
& +\left(\delta\left\langle\Psi_{i}\right|\right)\left\langle\Upsilon|\exp (\mathrm{i} g A \otimes \Omega)| \Psi_{f}\right\rangle\left(\Delta M\left|\Upsilon_{f}\right\rangle-\left|\Upsilon_{f}\right\rangle\langle\Delta M\rangle_{\mathrm{e}}\right) \\
& + \text { c.c. }=0 .
\end{aligned}
$$

Note that the variations $\delta\left|\Psi_{i}\right\rangle$ and $\delta\left|\Psi_{f}\right\rangle$ are arbitrary and independent, it follows from (7) that

$$
\begin{gathered}
\left\langle\Upsilon|\exp (\mathrm{i} g A \otimes \Omega)| \Psi_{f}\right\rangle\left(\Delta M-\langle\Delta M\rangle_{\mathrm{e}}\right)\left|\Upsilon_{f}\right\rangle=0 \\
\langle\Upsilon|\left\langle\Psi_{i}\left|\exp (\mathrm{i} g A \otimes \Omega)\left(\Delta M-\langle\Delta M\rangle_{\mathrm{e}}\right)\right| \Upsilon_{f}\right\rangle=0 .
\end{gathered}
$$

It is crucial to observe that the left sides of the two equations in (8) are vectors in the system Hilbert space, so their amplitudes in the eigenbasis of $A$ must all be zero. Suppose the observable $A$ has $r_{A}$ eigenvalues $a_{1}, \cdots, a_{d_{\mathrm{s}}}$ with eigenstates $\left|a_{1}\right\rangle, \cdots,\left|a_{d_{\mathrm{s}}}\right\rangle$. If $\left|\Psi_{i}\right\rangle=$ $\sum_{k} \alpha_{k}\left|a_{k}\right\rangle,\left|\Psi_{f}\right\rangle=\sum_{k} \beta_{k}\left|a_{k}\right\rangle$, the two equations of (8) give

$$
\begin{aligned}
& \sum_{k=1}^{d_{\mathrm{s}}} \alpha_{k}\left|a_{k}\right\rangle\left\langle\Upsilon\left|\exp \left(\mathrm{i} g a_{k} \Omega\right)\left(\Delta M-\langle\Delta M\rangle_{\mathrm{e}}\right)\right| \Upsilon_{f}\right\rangle=0, \\
& \sum_{k=1}^{d_{\mathrm{s}}} \beta_{k}^{*}\left\langle a_{k}\right|\left\langle\Upsilon\left|\exp \left(\mathrm{i} g a_{k} \Omega\right)\left(\Delta M-\langle\Delta M\rangle_{\mathrm{e}}\right)\right| \Upsilon_{f}\right\rangle=0 .
\end{aligned}
$$

Since either $\left|\Psi_{i}\right\rangle$ or $\left|\Psi_{f}\right\rangle$ can be arbitrary, we can choose all $\alpha_{k} \neq 0$ or all $\beta_{k} \neq 0$. Then the above equation shows

$$
\left\langle\Upsilon\left|\exp \left(\mathrm{i} g a_{i} \Omega\right)\left(\Delta M-\langle\Delta M\rangle_{\mathrm{e}}\right)\right| \Upsilon_{f}\right\rangle=0
$$

for all $i=1, \cdots, r_{A}$.

Let $\Xi_{g}$ be the matrix $\left[\mathrm{e}^{-\mathrm{i} g a_{1} \Omega}|\Upsilon\rangle|\cdots| \mathrm{e}^{-\mathrm{i} g a_{d_{\mathrm{s}}} \Omega}|\Upsilon\rangle\right]$. We can write $\left|\Upsilon_{f}\right\rangle$ as a linear combination of $\exp \left(-\mathrm{i} g a_{k} \Omega\right)|\Upsilon\rangle, k=1, \cdots, d_{\text {s }}$, i.e., $\left|\Upsilon_{f}\right\rangle=\Xi_{g} \boldsymbol{\mu}, \boldsymbol{\mu}=$ $\left(\mu_{1}, \cdots, \mu_{d_{\mathrm{s}}}\right)^{T}$, and $\mu_{k}=\beta_{k}^{*} \alpha_{k}$. Then

$$
\left(\Xi_{g}^{\dagger} \Delta M \Xi_{g}-\Xi_{g}^{\dagger} \Xi_{g}\langle\Delta M\rangle_{\mathrm{e}}\right) \boldsymbol{\mu}=0,
$$

Eq. (11) is a homogeneous linear equation with respect to $\boldsymbol{\mu}$, so the necessary and sufficient condition for the existence of nonzero $\boldsymbol{\mu}$ is

$$
\operatorname{det}\left(\Xi_{g}^{\dagger} \Delta M \Xi_{g}-\Xi_{g}^{\dagger} \Xi_{g}\langle\Delta M\rangle_{\mathrm{e}}\right)=0
$$

which is the major equation that $\langle\Delta M\rangle_{\mathrm{e}}$ must satisfy.

Eq. (12) implies that an extremum of $\langle\Delta M\rangle$ must be an eigenvalue of $\left(\Xi_{g}^{\dagger} \Xi_{g}\right)^{-\frac{1}{2}} \Xi_{g}^{\dagger} \Delta M \Xi_{g}\left(\Xi_{g}^{\dagger} \Xi_{g}\right)^{-\frac{1}{2}}$. Therefore, the largest $\langle\Delta M\rangle_{\mathrm{e}}$ is its largest eigenvalue:

$$
|\langle\Delta M\rangle|_{\max }=\left|\lambda\left(\left(\Xi_{g}^{\dagger} \Xi_{g}\right)^{-\frac{1}{2}} \Xi_{g}^{\dagger} \Delta M \Xi_{g}\left(\Xi_{g}^{\dagger} \Xi_{g}\right)^{-\frac{1}{2}}\right)\right|_{\max } .
$$

Note that above we have assumed $\Xi_{g}$ to be full rank, so that $\left(\Xi_{g}^{\dagger} \Xi_{g}\right)^{\frac{1}{2}}$ is invertible. If the eigenvalues of $A$ are degenerate, or $d_{\mathrm{s}}>d_{\mathrm{D}}$, the rank of $\Xi_{g}$ will be less than $d_{\mathrm{s}}$; in that case, one needs to pick out a maximal linearly independent subset from $\exp \left(-\mathrm{i} g a_{i} \Omega\right)|\Upsilon\rangle, i=1, \cdots, d_{\mathrm{s}}$ to construct the matrix $\Xi_{g}$.

A formal asymptotic solution for the amplification limit can be obtained from (13) by Gelfand's theorem [36] which connects the spectral radius of a matrix to its (arbitrary) norm. If we choose the norm $\|\cdot\|$ to be the trace norm, then

$$
|\langle\Delta M\rangle|_{\max }=\lim _{n \rightarrow \infty}\left(\operatorname{tr}\left(\Xi_{g}^{\dagger} \Delta M \Xi_{g}\left(\Xi_{g}^{\dagger} \Xi_{g}\right)^{-1}\right)^{n}\right)^{\frac{1}{n}}
$$

Usually, one can choose a finite $k$ to derive an approximate solution to $|\langle\Delta M\rangle|_{\max }$ from (14), and large $k$ will give higher precision to the approximation.

In a weak measurement, the coupling strength $g$ is usually very small. This can lead to a simplified and $g$ independent form of (12), which is helpful in finding the essential factors that determine the amplification limit.

The key is to prove that when $g \ll 1$, the support of $\Xi_{g}$, i.e., the subspace spanned by all translated detector states $\exp \left(-\mathrm{i} g a_{k} \Omega\right)|\Upsilon\rangle, k=1, \cdots, d_{\mathrm{s}}$, is $g$-independent, and can be spanned approximately by $|\Upsilon\rangle, \Omega|\Upsilon\rangle, \cdots, \Omega^{r_{A}-1}|\Upsilon\rangle$ (unorthonormalized), where $r_{A}$ is the number of different eigenvalues of $A$. The proof is given in Appendix A. If we define $\widetilde{\Xi}$ to be the matrix $\left.[|\Upsilon\rangle|\Omega| \Upsilon\rangle|\cdots| \Omega^{r_{A}-1}|\Upsilon\rangle\right]$, the equation for $\langle\Delta M\rangle_{\mathrm{e}}$ (12) can be simplified to

$$
\operatorname{det}\left(\widetilde{\Xi}^{\dagger} \Delta M \widetilde{\Xi}^{\widetilde{\Xi}}-\widetilde{\Xi}^{\dagger} \widetilde{\Xi}\langle\Delta M\rangle_{\mathrm{e}}\right)=0
$$

If $\widetilde{\Xi}$ is full rank, Eq. (15) is equivalent to the eigenvalue equation for $\left(\widetilde{\Xi}^{\dagger} \widetilde{\Xi}\right)^{-\frac{1}{2}} \widetilde{\Xi}^{\dagger} \Delta M \widetilde{\Xi}\left(\widetilde{\Xi} \widetilde{\Xi}^{\dagger} \widetilde{\Xi}\right)^{-\frac{1}{2}}$, so

$$
|\langle\Delta M\rangle|_{\max }=\left|\lambda\left(\left(\widetilde{\Xi}^{\dagger} \widetilde{\Xi}\right)^{-\frac{1}{2}} \widetilde{\Xi}^{\dagger} \Delta M \widetilde{\Xi}\left(\widetilde{\Xi}^{\dagger} \widetilde{\Xi}\right)^{-\frac{1}{2}}\right)\right|_{\max } \cdot
$$

If the rank of $\widetilde{\Xi}$ is less than $r_{A}$, one just needs to pick out a maximal linearly independent set from $|\Upsilon\rangle, \Omega|\Upsilon\rangle, \cdots, \Omega^{r_{A}-1}|\Upsilon\rangle$ to reconstruct $\widetilde{\Xi}$. So (16) will still hold.

Eq. (16) is a general solution for the amplification limit $|\langle\Delta M\rangle|_{\max }$. An explicit asymptotic solution can also be derived from (16) by Gelfand's theorem [36], which connects the spectral radius of a matrix to its (arbitrary) norm. If we choose the norm $\|\cdot\|$ in [36] to be the trace norm, then

$$
|\langle\Delta M\rangle|_{\max }=\lim _{n \rightarrow \infty}\left(\operatorname{tr}\left(\widetilde{\Xi}^{\dagger} \Delta M \widetilde{\Xi}\left(\widetilde{\Xi}^{\dagger} \widetilde{\Xi}\right)^{-1}\right)^{n}\right)^{\frac{1}{n}}
$$


We see from (16) and (17) that the amplification limit $|\langle\Delta M\rangle|_{\max }$ is independent of $g$, and determined only by the subspace spanned by $|\Upsilon\rangle, \Omega|\Upsilon\rangle, \cdots, \Omega^{r_{A}-1}|\Upsilon\rangle$. Denote this subspace as $\mathcal{H}_{\mathrm{D}}$. Usually, when $r_{A}$ increases, $\mathcal{H}_{\mathrm{D}}$ will become larger, so $|\langle\Delta M\rangle|_{\max }$ will increase as well. But if $r_{A}>r_{\Omega}$, where $r_{\Omega}$ is the number of distinct eigenvalues of $\Omega$, then this subspace will be $r_{\Omega}$ dimensional at most, and the increase of $r_{A}$ will no longer affect $|\langle\Delta M\rangle|_{\text {max }}$. In addition, the size of the support of $|\Upsilon\rangle$ on the eigenbasis of $\Omega$ also influences this subspace. For example, if $|\Upsilon\rangle$ is an eigenstate of $\Omega$, then $\mathcal{H}_{\mathrm{D}}$ is one dimensional, whatever $r_{A}$ and $r_{\Omega}$ are. A detailed analysis is given in Appendix B.

\section{EXAMPLES}

\section{A. Spin- $\frac{1}{2}$ particles}

To illustrate our result, we consider an example of spin$\frac{1}{2}$ particles with a pair of Stern-Gerlach devices [1].

When a beam of spin- $\frac{1}{2}$ particles with the same spin direction moving in the $x$ direction pass through a SternGerlach device which has a nonuniform external magnetic field in the $z$ direction, it will be coupled to the magnetic field due to the interaction

$$
H_{I}=-\mu \frac{\partial B_{z}}{\partial z} z \sigma_{z} \delta\left(x-x_{0}\right),
$$

where $\mu$ is the magnetic moment of a single particle, and $\delta\left(x-x_{0}\right)$ means that the support of the magnetic field is very narrow so that the duration of the interaction is extremely short. Immediately after the first Stern-Gerlach device, let the beam of particles pass through a second Stern-Gerlach device, where the magnetic field is in the $y$ direction. Then the particles will split into two beams with spins pointing to the $\pm y$ directions respectively. If we keep track of one of the two beams, say the beam with spins $+y$, then we actually postselect the particles in the state of spin $+y$. If the gradient of the magnetic field in the first Stern-Gerlach device is sufficiently small, i.e. $\left|\frac{\partial B_{z}}{\partial z}\right| \ll 1$, and the initial direction of the spins is properly chosen to be close to the $-y$ direction, then the $+y$ beam will have a large displacement in the $z$ direction due to the amplification effect of the postselected weak measurement.

Now, we can apply the results derived above to find the largest possible displacement of the postselected beam over all directions of the magnetic field in the second Stern-Gerlach device. In this example, $g=-\mu \frac{\partial B_{z}}{\partial z}, \Omega=$ $z, M=z, p_{z}$. Suppose the initial spatial wave function of the particles in the $z$ direction is $\Upsilon(z)$ and is symmetric about its center for simplicity. Since $d_{\mathrm{s}}=2$ for spin- $\frac{1}{2}$ particles, the final spatial wave function of the particles can be spanned by $\Upsilon(z)$ and $z \Upsilon(z)$ (unnormalized). If the position or the momentum of the particle is measured in the $z$ direction after the second Stern-Gerlach device,

\begin{tabular}{cccc}
\hline Detector state & Detector wave function & $|\langle\Delta z\rangle|_{\max }$ & $\left.\left\langle\Delta p_{z}\right\rangle\right|_{\max }$ \\
\hline \hline Gaussian & $\left((2 \pi)^{\frac{1}{2}} K\right)^{-\frac{1}{2}} \exp \left(-\frac{z^{2}}{4 K^{2}}\right)$ & $K$ & $\frac{1}{2} K^{-1}$ \\
\hline Lorentzian & $\left(\frac{\pi K}{2}\right)^{-\frac{1}{2}} \frac{1}{1+(z / K)^{2}}$ & $K$ & $\frac{1}{2} K^{-1}$ \\
\hline Exponential & $K^{-\frac{1}{2}} \exp \left(-\frac{|z|}{K}\right)$ & $\frac{1}{\sqrt{2}} K$ & $\frac{1}{\sqrt{2}} K^{-1}$ \\
\hline
\end{tabular}

Table I. Results for three typical examples with $d_{\mathrm{s}}=2$. The maximal position shift $|\langle\Delta q\rangle|_{\max }$ can be very large for all three examples if the spread width of the states (proportional to $K$ ) is sufficiently large, or the maximal momentum shift $|\langle\Delta p\rangle|_{\max }$ can be large if $K$ is sufficiently small. This also verifies the complementarity relation (20).

then from Eq. (16) one can obtain (see Appendix C)

$$
\begin{aligned}
|\langle\Delta z\rangle|_{\max } & =\sqrt{\left\langle\Delta z^{2}\right\rangle_{\Upsilon}}, \\
\left|\left\langle\Delta p_{z}\right\rangle\right|_{\max } & =\frac{1}{2} \sqrt{\frac{1+\left\langle\left\{z, \Delta p_{z}\right\}\right\rangle_{\Upsilon}^{2}}{\left\langle\Delta z^{2}\right\rangle_{\Upsilon}}},
\end{aligned}
$$

where $\langle\Upsilon|\cdot| \Upsilon\rangle$ is denoted $\langle\cdot\rangle_{\Upsilon}$ for brevity. From (19), one can see that neither $|\langle\Delta z\rangle|_{\max }$ nor $\left|\left\langle\Delta p_{z}\right\rangle\right|_{\max }$ depend on the magnetic moment $\mu$ or the external field $B_{z}$, and they are determined only by the initial wave function of the particle, which confirms the previous result.

We applied (19) to three typical spatial wave functions for the particles: the Gaussian state, the Lorentzian state and the exponential state; the results are summarized in Table I. In addition, the results for the exponential state are plotted in Fig. I for different weak values. It can be seen that upper bounds always exist whatever $g$ is, and the upper bounds are nearly the same when $g \ll 1$, which verifies the previous result.

An important complementarity relation between $|\langle\Delta z\rangle|_{\max }$ and $\left|\left\langle\Delta p_{z}\right\rangle\right|_{\max }$ can be obtained from (19):

$$
|\langle\Delta z\rangle|_{\max }\left|\left\langle\Delta p_{z}\right\rangle\right|_{\max }=\frac{1}{2} \sqrt{1+\left\langle\left\{z, \Delta p_{z}\right\}\right\rangle_{\Upsilon}^{2}} \geq \frac{1}{2} .
$$

If the wave function $\Upsilon(z)$ is real, then $\left\langle\left\{z, \Delta p_{z}\right\}\right\rangle_{\Upsilon}=$ 0 , so the complementarity relation becomes an equality: $|\langle\Delta z\rangle|_{\max }\left|\left\langle\Delta p_{z}\right\rangle\right|_{\max }=\frac{1}{2}$.

According to the Robertson-Schrödinger uncertainty inequality [37], the second equation of (19) leads to $\left|\left\langle\Delta p_{z}\right\rangle\right|_{\max } \leq \sqrt{\left\langle\Delta z^{2}\right\rangle_{\Upsilon}}$, so it can be combined with the second equation of (19) as

$$
|\langle\Delta M\rangle|_{\max } \leq \sqrt{\left\langle\Delta M^{2}\right\rangle_{\Upsilon}}, M=z, p_{z}
$$

The upper bound of the amplification limit (21) has an intuitive physical picture: the final spatial wave function of a single particle is a superposition of several wave function that are translated very little from the initial wave function. When the postselection on the particle's spin is properly chosen, the superposition can cancel the major part of the initial wave function, resulting in a large deviation of the particle's spatial wave function from its original position. But such a displacement cannot be too large, because the wave function is still bounded. So the 


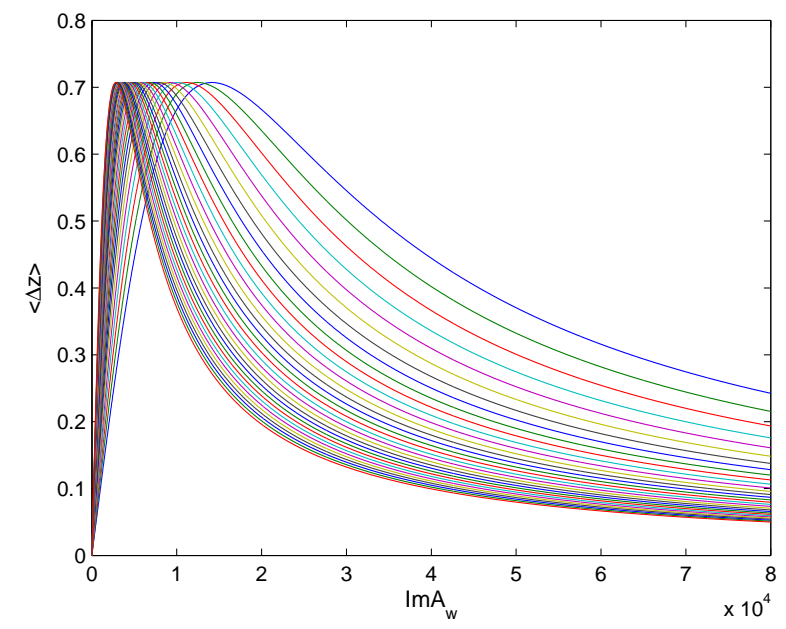

Figure 1. (Color online) The figure shows the average shift of spin- $\frac{1}{2}$ particles $\langle\Delta z\rangle$ as $\operatorname{Im} A_{w}$ increases. The particles are in an exponential state $\exp (-|q|)$ initially, and the Hamiltonian is (18). Different curves are plotted for different $-\mu \frac{\partial B_{z}}{\partial z}$, ranging from $10^{-4}$ to $5 \times 10^{-4}$ (steeper curves with larger $\left.-\mu \frac{\partial B_{z}}{\partial z}\right)$. Explicit turning points can be found in this figure, which indicates the upper bound of the amplification effect. It can be seen that the largest $\langle\Delta z\rangle$ are almost the same for different $-\mu \frac{\partial B_{z}}{\partial z}$, which confirms the independence from $g$ of $|\langle\Delta M\rangle|_{\max }$.

particle can be only shifted to the edge of the spread of its wave function at most. This is what Eq. (19) implies.

The above results can help to choose appropriate settings in designing real weak measurement experiments. The initial detector states can be chosen by (19) or (21) to realize a desired amplification limit. For example, if one wants to enlarge the maximal position shift of the detector, one should choose an initial state with wider spread for the detector; to enlarge the maximal momentum shift of the detector, the initial spread of the detector should be narrower. The width of the initial detector wave function decides the amplification limit of a weak measurement with that detector.

\section{B. Continuous variable systems}

The role of the dimension of the system is often neglected in the study of weak measurement amplification. To show how the dimension of the system can influence the amplification limit, we now consider continuous variable systems, i.e., $d_{\mathrm{s}}=\infty$, as a limiting case of high dimensional systems. For simplicity, we assume $A$ and $\Omega$ are nondegenerate, i.e. $r_{A}=d_{\mathrm{s}}$ and $r_{\Omega}=d_{\mathrm{D}}$.

When $d_{\mathrm{s}}=\infty$, the subspace $\mathcal{H}_{\mathrm{D}}$ of all possible final detector states can be spanned by $|\Upsilon\rangle, \Omega|\Upsilon\rangle, \cdots, \Omega^{\infty}|\Upsilon\rangle$. Obviously, this subspace is equal to the whole Hilbert space of the detector only if $|\Upsilon\rangle$ has full support on the eigenbasis of $\Omega$. This implies that the final detector can be (but is not limited to) any eigenstate of $M$. Therefore, $|\langle\Delta M\rangle|_{\max }=|\lambda(M)|_{\max }$ in this case. In particular, if the detector is also a continuous variable system and $M=q, p$, then $|\langle\Delta M\rangle|_{\max }=\infty$, but is not bounded by the spread of the detector state $\sqrt{\left\langle\Delta M^{2}\right\rangle_{\Upsilon}}$ as in (21), a dramatic difference from the results for $d_{\mathrm{s}}=2$.

Of course, one cannot always postselect a continuous variable system to be in an arbitrary state, so $|\langle\Delta M\rangle|_{\max }$ will still be finite, or only approach $\infty$ asymptotically, in practice. Yet this limiting example demonstrates how significantly the dimension of the system can influence the amplification limit of a weak measurement, particularly when the system dimension is high, which clearly shows the advantage of the variational method, since such a result cannot be derived from the first-order weak value formalism [33]. And it reveals the rich structure and complexity of this problem.

\section{GENERALIZATION TO MIXED DETECTOR STATES}

In this section, we generalize the main results to mixed detector states. Suppose the initial state of the detector is mixed:

$$
\rho_{\mathrm{D}}=\sum_{k} \eta_{k}\left|\Upsilon_{k}\right\rangle\left\langle\Upsilon_{k}\right|
$$

The generalization to this case is mostly straightforward, but one must take care to avoid a tricky pitfall. At first glance, as $\rho_{\mathrm{D}}$ represents the ensemble $\left\{\eta_{k},\left|\Upsilon_{k}\right\rangle\right\}$, it seems that the maximum shift of the detector should be the average maximum shift of the detector over the ensemble:

$$
|\langle\Delta M\rangle|_{\max } \stackrel{?}{=} \sum_{k} \eta_{k}|\langle\Delta M\rangle|_{\max }^{\left|\Upsilon_{k}\right\rangle}
$$

where the superscript $\left|\Upsilon_{k}\right\rangle$ indicates the dependence of $|\langle\Delta M\rangle|_{\max }$ on $\left|\Upsilon_{k}\right\rangle$. But the optimal choice of pre- and postselections to reach the maximum shift depends on the initial detector state, so for different $\left|\Upsilon_{k}\right\rangle$ 's, the optimal choices of pre- and postselections are different. One cannot make the optimal choice for all $\left|\Upsilon_{k}\right\rangle$ 's simultaneously. So (23) is not the maximal shift for a mixed detector state, in general, but rather an upper bound on the shift.

In fact, by carrying out the previous variational procedure for mixed detector states, it can be shown that $|\langle\Delta M\rangle|_{\max }$ is the largest absolute value over all solutions to

$$
\operatorname{det} \sum_{k} \eta_{k}\left(\widetilde{\Xi}_{k}^{\dagger} \Delta M \widetilde{\Xi}_{k}-\widetilde{\Xi}_{k}^{\dagger} \widetilde{\Xi}_{k}\langle\Delta M\rangle_{\mathrm{e}}\right)=0
$$

where $\widetilde{\Xi}_{k}$ is the matrix with $\left|\Upsilon_{k}\right\rangle, \Omega\left|\Upsilon_{k}\right\rangle, \cdots, \Omega^{d_{\mathrm{s}}-1}\left|\Upsilon_{k}\right\rangle$ as its columns, as defined above. So the maximum detector shift is

$$
\begin{aligned}
|\langle\Delta M\rangle|_{\max } & =\mid \lambda\left(\left(\sum_{i} \eta_{i} \widetilde{\Xi}_{i}^{\dagger} \widetilde{\Xi}_{i}\right)^{-\frac{1}{2}} \sum_{k} \eta_{k} \widetilde{\Xi}_{k}^{\dagger} \Delta M\right. \\
& \left.\times \widetilde{\Xi}_{k}\left(\sum_{j} \eta_{j} \widetilde{\Xi}_{j}^{\dagger} \widetilde{\Xi}_{j}\right)^{-\frac{1}{2}}\right)\left.\right|_{\max },
\end{aligned}
$$


and an asymptotic solution is

$$
\begin{aligned}
& |\langle\Delta M\rangle|_{\max } \\
= & \lim _{n \rightarrow \infty}\left(\operatorname{tr}\left(\sum_{k} \eta_{k} \widetilde{\Xi}_{k}^{\dagger} \Delta M \widetilde{\Xi}_{k}\left(\sum_{j} \eta_{j} \widetilde{\Xi}_{j}^{\dagger} \widetilde{\Xi}_{j}\right)^{-\frac{1}{2}}\right)^{n}\right)^{\frac{1}{n}} .
\end{aligned}
$$

\section{ACKNOWLEDGMENTS}

Shengshi Pang thanks Justin Dressel and Antonio Di Lorenzo for helpful discussions. Shengshi Pang and Todd A. Brun acknowledge support from the ARO MURI under Grant No. W911NF-11-1-0268 and the NSF grant CCF-0829870. Shengjun Wu thanks support from the NSFC under Grant No. 11275181. Zeng-Bing Chen thanks support from NNSF of China under Grant No. 61125502, the CAS, the National High Technology Research and Development Program of China, and the National Fundamental Research Program under Grant No. 2011CB921300.

\section{APPENDIX A. THE SUBSPACE OF THE FINAL DETECTOR STATES}

In this appendix, we prove by induction that $|\Upsilon\rangle, \Omega|\Upsilon\rangle, \cdots, \Omega^{\left(r_{A}-1\right)}|\Upsilon\rangle \quad$ is an approximate (unorthonormalized) spanning set for the subspace spanned by the translated detector states $\exp \left(-\mathrm{i} g a_{k} \Omega\right)|\Upsilon\rangle, k=1, \cdots, r_{A}$, when $g|\lambda(A)|_{\max } \ll 1$.

Suppose that $A$ has $r_{A}$ distinct eigenvalues. The subspace of final detector states, which we denote as $\mathcal{H}_{\mathrm{D}}$, can be approximately spanned by the vectors $\left\{\exp \left(-\mathrm{i} g a_{k} \Omega\right)|\Upsilon\rangle\right\}, k=1, \cdots, r_{A}$. Define the matrix

$$
\left.\widetilde{\Xi}=[|\Upsilon\rangle|\Omega| \Upsilon\rangle|\cdots| \Omega^{r_{A}-1}|\Upsilon\rangle\right]
$$

It is easy to verify that the columns of $\widetilde{\Xi}\left(\widetilde{\Xi}^{\dagger} \widetilde{\Xi}\right)^{-\frac{1}{2}}$ are an orthonormal basis of $\mathcal{H}_{\mathrm{D}}$.

Now suppose that $A$ has $r_{A}+1$ distinct eigenvalues, and we have constructed the matrix $\widetilde{\Xi}$ as above using the first $r_{A}$ eigenvalues. By the Gram-Schmidt orthogonalization procedures, the next state in the basis (if there is one) can be obtained by

$$
\begin{aligned}
\left|e_{r_{A}+1}\right\rangle & =\exp \left(-\mathrm{i} g a_{r_{A}+1} \Omega\right)|\Upsilon\rangle \\
& -\widetilde{\Xi}\left(\widetilde{\Xi}^{\dagger} \widetilde{\Xi}\right)^{-\frac{1}{2}}\left(\widetilde{\Xi}^{\dagger} \widetilde{\Xi}\right)^{-\frac{1}{2}} \widetilde{\Xi}^{\dagger} \exp \left(-\mathrm{i} g a_{r_{A}+1} \Omega\right)|\Upsilon\rangle \\
& =\exp \left(-\mathrm{i} g a_{r_{A}+1} \Omega\right)|\Upsilon\rangle \\
& -\widetilde{\Xi}\left(\widetilde{\Xi}^{\dagger} \widetilde{\Xi}\right)^{-1} \widetilde{\Xi}^{\dagger} \exp \left(-\mathrm{i} g a_{r_{A}+1} \Omega\right)|\Upsilon\rangle .
\end{aligned}
$$

Note that we assumed $\underset{\Xi}{\widetilde{\Xi}}$ to have a full rank so that $\underset{\Xi}{\widetilde{\Xi}} \widetilde{\Xi}$ is invertible in (28). If $\widetilde{\Xi}$ does not have a full rank, then one should use the pseudoinverse of $\widetilde{\Xi}^{\dagger} \widetilde{\Xi}$ (the inverse on its support) instead.
Since $g|\lambda(A)|_{\max } \ll 1$,

$$
\begin{aligned}
& \exp \left(-\mathrm{i} g a_{r_{A}+1} \Omega\right)|\Upsilon\rangle \\
= & \sum_{k=0}^{r_{A}} \frac{\left(-\mathrm{i} g a_{r_{A}+1}\right)^{k}}{k !} \Omega^{k}|\Upsilon\rangle+o\left(g^{r_{A}}\right) \\
= & \widetilde{\Xi} X+\frac{\left(-\mathrm{i} g a_{r_{A}+1}\right)^{r_{A}}}{r_{A} !} \Omega^{r_{A}}|\Upsilon\rangle+o\left(g^{r_{A}}\right),
\end{aligned}
$$

where

$$
X=\left(1,-\mathrm{i} g a_{r_{A}+1}, \cdots, \frac{\left(-\mathrm{i} g a_{r_{A}+1}\right)^{r_{A}-1}}{\left(r_{A}-1\right) !}\right)^{T} .
$$

Therefore, (28) can be simplified to

$$
\begin{aligned}
& \left|e_{r_{A}+1}\right\rangle \\
= & \widetilde{\Xi} X+\frac{\left(-\mathrm{i} g a_{r_{A}+1}\right)^{r_{A}}}{r_{A} !} \Omega^{r_{A}}|\Upsilon\rangle+o\left(g^{r_{A}}\right) \\
& -\widetilde{\Xi}\left(\widetilde{\Xi}^{\dagger} \widetilde{\Xi}\right)^{-1} \widetilde{\Xi}^{\dagger}\left(\widetilde{\Xi} X+\frac{\left(-\mathrm{i} g a_{r_{A}+1}\right)^{r_{A}}}{r_{A} !} \Omega^{r_{A}}|\Upsilon\rangle+o\left(g^{r_{A}}\right)\right) \\
= & \frac{\left(-\mathrm{i} g a_{r_{A}+1}\right)^{r_{A}}}{r_{A} !}\left(\Omega^{r_{A}}|\Upsilon\rangle-\widetilde{\Xi}\left(\widetilde{\Xi}^{\dagger} \widetilde{\Xi}\right)^{-1} \widetilde{\Xi}^{\dagger} \Omega^{r_{A}}|\Upsilon\rangle\right)+o\left(g^{r_{A}}\right) .
\end{aligned}
$$

Since the columns of $\widetilde{\Xi}$ are $|\Upsilon\rangle, \Omega|\Upsilon\rangle, \cdots, \Omega^{r_{A}-1}|\Upsilon\rangle$, it can be seen from (31) that $\left|e_{r_{A}+1}\right\rangle$ is a linear combination of $|\Upsilon\rangle, \Omega|\Upsilon\rangle, \cdots, \Omega^{r_{A}}|\Upsilon\rangle$. Thus, the subspace spanned by $\exp \left(-\mathrm{i} g a_{k} \Omega\right)|\Upsilon\rangle, k=1, \cdots, r_{A}+1$ can be spanned by $|\Upsilon\rangle, \Omega|\Upsilon\rangle, \cdots, \Omega^{r_{A}}|\Upsilon\rangle$. This completes the proof by induction.

\section{APPENDIX B. ANALYSIS OF THE DIMENSION OF $\mathcal{H}_{\mathrm{D}}$}

In this appendix, we analyze the dimension of the subspace $\mathcal{H}_{\mathrm{D}}$ spanned by all possible final detector states, so as to show what determines the amplification limit $|\langle\Delta M\rangle|_{\max }$. The vectors $|\Upsilon\rangle, \Omega|\Upsilon\rangle, \cdots, \Omega^{r_{A}-1}|\Upsilon\rangle$ can be chosen to be a spanning set for $\mathcal{H}_{\mathrm{D}}$, as proved in Appendix $\mathrm{A}$, so the dimension of $\mathcal{H}_{\mathrm{D}}$ is equal to the rank of the matrix $\left.\widetilde{\Xi}=[|\Upsilon\rangle|\Omega| \Upsilon\rangle|\cdots| \Omega^{r_{A}-1}|\Upsilon\rangle\right]$.

Let $\Omega$ have $r_{\Omega}$ distinct eigenvalues $\omega_{1}, \cdots, \omega_{r_{\Omega}}$, and let the projectors of the corresponding eigensubspaces be $P_{\omega_{k}}, k=1, \cdots, r_{\Omega}$. Then $|\Upsilon\rangle=\sum_{k=1}^{r \Omega} P_{\omega_{k}}|\Upsilon\rangle$, then $\Omega^{i}|\Upsilon\rangle=\sum_{k=1}^{r_{\Omega}} \omega_{k}^{i} P_{\omega_{k}}|\Upsilon\rangle$, and $\widetilde{\Xi}=C D$, where

$$
C=\left(\begin{array}{cccc}
c_{1} & & & \\
& c_{2} & & \\
& & \ddots & \\
& & & c_{r_{\Omega}}
\end{array}\right), D=\left(\begin{array}{cccc}
1 & \omega_{1} & \cdots & \omega_{1}^{r_{A}-1} \\
1 & \omega_{2} & \cdots & \omega_{2}^{r_{A}-1} \\
\vdots & \vdots & \ddots & \vdots \\
1 & \omega_{r_{\Omega}} & \cdots & \omega_{r_{\Omega}}^{r_{A}-1}
\end{array}\right)
$$

The computational basis is $\frac{P_{\omega_{k}}|\Upsilon\rangle}{\sqrt{\left\langle\Upsilon\left|P_{\omega_{k}}\right| \Upsilon\right\rangle}}$, and $c_{k}=$ $\sqrt{\left\langle\Upsilon\left|P_{\omega_{k}}\right| \Upsilon\right\rangle}$

When $|\Upsilon\rangle$ has a full support on the eigenbasis of $\Omega$, i.e. $c_{k} \neq 0$ for all $k=1, \cdots, r_{\Omega}, \operatorname{dim}\left(\mathcal{H}_{\mathrm{D}}\right)=\operatorname{rank}(\widetilde{\Xi})=$ 
$\operatorname{rank}(D)$. Since $\omega_{1}, \cdots, \omega_{r_{\Omega}}$ are distinct from each other, it can be inferred that

$$
\operatorname{dim}\left(\mathcal{H}_{\mathrm{D}}\right)= \begin{cases}r_{A}, & r_{A} \leq r_{\Omega} \\ r_{\Omega}, & r_{A}>r_{\Omega}\end{cases}
$$

When $|\Upsilon\rangle$ does not have full support on the eigenbasis of $\Omega$, say $c_{n+1}=\cdots=c_{r_{\Omega}}=0$, then similarly to the above equation, it can be shown that

$$
\operatorname{dim}\left(\mathcal{H}_{\mathrm{D}}\right)= \begin{cases}r_{A}, & r_{A} \leq n \\ n, & r_{A}>n\end{cases}
$$

In summary, the initial detector state $|\Upsilon\rangle$, the number of distinct eigenvalues $r_{A}$ and $r_{\Omega}$ of $A$ and $\Omega$ (respectively), and the support of $|\Upsilon\rangle$ in the eigenbasis of $\Omega$ determine the amplification limit.

\section{APPENDIX C. GENERAL DISCUSSION FOR

$$
d_{\mathrm{s}}=2
$$

In the main text, we showed an example of spin- $\frac{1}{2}$ particles passing through two Stern-Gerlach devices to illustrate the main results. In this appendix, we want to give a general discussion about the results for two dimensional systems, which is of great interest in the field of quantum information and quantum computing. We assume $A$ to be nondegenerate, since otherwise $A$ would be proportional to the identity and the interaction would be trivial.

\section{A. General results for $d_{\mathrm{s}}=2$.}

When $d_{\mathrm{s}}=2$, the subspace spanned by all possible final detector states is approximately spanned by $|\Upsilon\rangle$ and $\Omega|\Upsilon\rangle$. We assume that $|\Upsilon\rangle$ is not an eigenstate of $\Omega$ so that $|\Upsilon\rangle$ and $\Omega|\Upsilon\rangle$ are linearly independent. We shall consider three typical cases below. In this section, we shall denote $\langle\Upsilon|\cdot| \Upsilon\rangle$ by $\langle\cdot\rangle_{\Upsilon}$ for short.

For $d_{\mathrm{s}}=2,\langle\Delta M\rangle_{\mathrm{e}}$ satisfies

$$
\begin{aligned}
& \operatorname{det}\left(\begin{array}{cc}
-\langle\Delta M\rangle_{\mathrm{e}} & \langle\Delta M \Omega\rangle_{\Upsilon}-\langle\Omega\rangle_{\Upsilon}\langle\Delta M\rangle_{\mathrm{e}} \\
\langle\Omega \Delta M\rangle_{\Upsilon}-\langle\Omega\rangle_{\Upsilon}\langle\Delta M\rangle_{\mathrm{e}} & \langle\Omega \Delta M \Omega\rangle_{\Upsilon}-\left\langle\Omega^{2}\right\rangle_{\Upsilon}\langle\Delta M\rangle_{\mathrm{e}}
\end{array}\right) \\
& =0,
\end{aligned}
$$

where we have used $\langle\Delta M\rangle_{\Upsilon}=0 . \quad|\langle\Delta M\rangle|_{\max }$ can be straightforwardly derived from the above equation:

$$
|\langle\Delta M\rangle|_{\max }=\frac{W_{\Upsilon}}{2\left\langle\Delta \Omega^{2}\right\rangle_{\Upsilon}}
$$

where $\left\langle\Delta \Omega^{2}\right\rangle_{\Upsilon}=\left\langle\Omega^{2}\right\rangle_{\Upsilon}-\langle\Omega\rangle_{\Upsilon}^{2}$, and

$$
\begin{aligned}
W_{\Upsilon} & =\left|\langle\Omega\rangle_{\Upsilon}\langle\{\Omega, \Delta M\}\rangle_{\Upsilon}-\langle\Omega \Delta M \Omega\rangle_{\Upsilon}\right| \\
& +\left(\left(\langle\Omega\rangle_{\Upsilon}\langle\{\Omega, \Delta M\}\rangle_{\Upsilon}-\langle\Omega \Delta M \Omega\rangle_{\Upsilon}\right)^{2}\right. \\
& \left.+\left\langle\Delta \Omega^{2}\right\rangle_{\Upsilon}\left(\langle\{\Omega, \Delta M\}\rangle_{\Upsilon}^{2}-\langle[\Omega, \Delta M]\rangle_{\Upsilon}^{2}\right)\right)^{\frac{1}{2}} .
\end{aligned}
$$

Note that $\langle\Delta M\rangle_{\Upsilon}=0$, so according to the RobertsonSchrödinger uncertainty inequality 37], an upper bound for $|\langle\Delta M\rangle|_{\max }$ is

$$
\begin{aligned}
|\langle\Delta M\rangle|_{\max } & \leq \frac{\left|\langle\Omega\rangle_{\Upsilon}\langle\{\Omega, \Delta M\}\rangle_{\Upsilon}-\langle\Omega \Delta M \Omega\rangle_{\Upsilon}\right|}{\left\langle\Delta \Omega^{2}\right\rangle_{\Upsilon}} \\
& +\sqrt{\left\langle\Delta M^{2}\right\rangle_{\Upsilon}},
\end{aligned}
$$

where $\left\langle\Delta M^{2}\right\rangle_{\Upsilon}=\left\langle M^{2}\right\rangle_{\Upsilon}-\langle M\rangle_{\Upsilon}^{2}$.

\section{B. Symmetric detector states}

Now, we assume that the initial detector state $|\Upsilon\rangle$ is symmetric about its center, and that $\Omega=q, M=q, p$. In this case, $\langle\Omega\rangle_{\Upsilon}=\langle\Omega \Delta M \Omega\rangle_{\Upsilon}=0$, so (38) implies that the maximal shift of the detector will not exceed the spread of the initial detector states in the eigenbasis of $M$. According to (36) the exact maximal shift of the detector should be

$|\langle\Delta q\rangle|_{\max }=\sqrt{\left\langle\Delta q^{2}\right\rangle_{\Upsilon}},|\langle\Delta p\rangle|_{\max }=\frac{1}{2} \sqrt{\frac{1+\langle\{q, \Delta p\}\rangle_{\Upsilon}^{2}}{\left\langle\Delta q^{2}\right\rangle_{\Upsilon}}}$.

An important property of $|\langle\Delta q\rangle|_{\max }$ and $|\langle\Delta p\rangle|_{\max }$ is that

$$
|\langle\Delta q\rangle|_{\max }|\langle\Delta p\rangle|_{\max }=\frac{1}{2} \sqrt{1+\langle\{q, \Delta p\}\rangle_{\Upsilon}^{2}} \geq \frac{1}{2},
$$

which indicates a complementarity relationship between $|\langle\Delta q\rangle|_{\max }$ and $|\langle\Delta p\rangle|_{\max }$. A special case is that when the wave function of the initial detector state is real, then $\langle\{q, \Delta p\}\rangle_{\Upsilon}=0$, and (40) becomes an equality:

$$
|\langle\Delta q\rangle|_{\max }|\langle\Delta p\rangle|_{\max }=\frac{1}{2}
$$

[1] Y. Aharonov, D. Z. Albert, and L. Vaidman, Phys. Rev. Lett. 60, 1351 (1988).

[2] I. M. Duck, P. M. Stevenson and E. C. G. Sudarshan,
Phys. Rev. D 40, 2112 (1989).

[3] O. Hosten and P. Kwiat, Science 319, 787 (2008).

[4] Y. Gorodetski, K. Y. Bliokh, B. Stein, C. Genet, N. 
Shitrit, V. Kleiner, E. Hasman, and T. W. Ebbesen, Phys. Rev. Lett. 109, 013901 (2012).

[5] P. B. Dixon, D. J. Starling, A. N. Jordan, and J. C. Howell, Phys. Rev. Lett. 102, 173601 (2009).

[6] D. J. Starling, P. B. Dixon, A. N. Jordan, and J. C. Howell, Phys. Rev. A 80, 041803 (2009).

[7] D. J. Starling, P. B. Dixon, A. N. Jordan, and J. C. Howell, Phys. Rev. A 82, 063822 (2010).

[8] D. J. Starling, P. B. Dixon, N. S. Williams, A. N. Jordan, and J. C. Howell, Phys. Rev. A 82, 011802(R) (2010).

[9] X.-Y. Xu, Y. Kedem, K. Sun, L. Vaidman, C.-F. Li, and G.-C. Guo, Phys. Rev. Lett. 111, 033604 (2013).

[10] P. Egan and J. A. Stone, Opt. Lett. 37, 4991 (2012).

[11] G. I. Viza, J. Martínez-Rincón, G. A. Howland, H. Frostig, I. Shomroni, B. Dayan, and J. C. Howell, Opt. Lett. 38, 2949 (2013).

[12] A. Romito and Y. Gefen, Phys. Rev. Lett. 100, 056801 (2008).

[13] V. Shpitalnik, Y. Gefen and A. Romito, Phys. Rev. Lett. 101, 226802 (2008).

[14] N. Brunner and C. Simon, Phys. Rev. Lett. 105, 010405 (2010).

[15] O. Zilberberg, A. Romito, and Y. Gefen, Phys. Rev. Lett. 106, 080405 (2011).

[16] S. Wu and M. Zukowski, Phys. Rev. Lett. 108, 080403 (2012).

[17] G. Strübi and C. Bruder, Phys. Rev. Lett. 110, 083605 (2013).

[18] M. Bula, K. Bartkiewicz, A. Černoch, and K. Lemr, Phys. Rev. A 87, 033826 (2013).

[19] E. Meyer-Scott, M. Bula, K. Bartkiewicz, A. Černoch, J. Soubusta, T. Jennewein, and K. Lemr, Phys. Rev. A 88, 012327 (2013).

[20] J. Dressel, K. Lyons, A. N. Jordan, T. M. Graham, and P. G. Kwiat, Phys. Rev. A 88, 023821 (2013).

[21] P. Campagne-Ibarcq, L. Bretheau, E. Flurin, A. Auffèves,
F. Mallet, and B. Huard, arXiv:1311.5605 (2013).

[22] J. P. Groen, D. Ristè, L. Tornberg, J. Cramer, P. C. de Groot, T. Picot, G. Johansson, and L. DiCarlo, Phys. Rev. Lett. 111, 090506 (2013).

[23] D. Lu, A. Brodutch, J. Li, H. Li, and R. Laflamme, arXiv:1311.5890 (2013).

[24] S. Wu and Y. Li, Phys. Rev. A 83, 052106 (2011).

[25] A. G. Kofman, S. Ashhab, F. Nori, Phys. Rep. 520, 43 (2012).

[26] J. Dressel, M. Malik, F. M. Miatto, A. N. Jordan, R. W. Boyd, Rev. Mod. Phys. 86, 307 (2014).

[27] A. Feizpour, X. Xing, and A. M. Steinberg, Phys. Rev. Lett. 107, 133603 (2011)

[28] T. Koike and S. Tanaka, Phys. Rev. A 84, 062106 (2011).

[29] K. Nakamura, A. Nishizawa, and M. K. Fujimoto, Phys. Rev. A 85, 012113 (2012).

[30] X. Zhu, Y. Zhang, S. Pang, C. Qiao, Q. Liu, and S. Wu, Phys. Rev. A 84, 052111 (2011).

[31] S. Pang, S. Wu, and Z.- B. Chen, Phys. Rev. A 86, 022112 (2012).

[32] Y. Susa, Y. Shikano, and A. Hosoya, Phys. Rev. A 85, 052110 (2012).

[33] A. D. Lorenzo, Ann. Phys. 345, 178 (2014).

[34] G. Puentes, N. Hermosa,1 and J. P. Torres, Phys. Rev. Lett. 109, 040401 (2012).

[35] R. Jozsa, Phys. Rev. A 76, 044103 (2007).

[36] Gelfand's theorem: for any matrix norm $\|\cdot\|$ and an arbitrary matrix $A$, the spectral radius of $A$ satisfies

$$
\rho(A)=\lim _{n \rightarrow \infty}\left\|A^{n}\right\|^{\frac{1}{n}} .
$$

[37] Robertson-Schrödinger uncertainty inequality: for any two Hermitian operators $\Omega_{1}$ and $\Omega_{2}$,

$\Delta \Omega_{1} \Delta \Omega_{2} \geq \frac{1}{2} \sqrt{\left|\left\langle\left[\Omega_{1}, \Omega_{2}\right]\right\rangle\right|^{2}+\left|\left\langle\left\{\Omega_{1}, \Omega_{2}\right\}\right\rangle-2\left\langle\Omega_{1}\right\rangle\left\langle\Omega_{2}\right\rangle\right|^{2}}$. 